\title{
Asymptotic Analysis for U-Statistics and Its Application to Von Mises Statistics
}

\author{
Timur Zubayraev \\ Faculty of Computational Mathematics and Cybernetics, Moscow State University, Moscow, Russia \\ E-mail: tzubayraev@gmail.com \\ Received July 30, 2011; revised August 31, 2011; accepted September 14, 2011
}

\section{Abstract}

Let $X, \bar{X}, X_{1}, \cdots, X_{N}$ be i.i.d. random variables taking values in a measurable space $(\mathfrak{X}, \mathfrak{B})$. Let $\phi_{1}: \mathfrak{X}$ $\rightarrow \mathbb{R}$ and $\phi: \mathfrak{X}^{2} \rightarrow \mathbb{R}$ be measurable functions. Assume that $\phi$ is symmetric, i.e. $\phi(x, y)=\phi(y, x)$, for any $x, y \in \mathfrak{X}$. Consider U-statistic $T=\frac{1}{N} \sum_{1 \leq i<j \leq N} \phi\left(X_{i}, X_{j}\right)+\frac{1}{\sqrt{N}} \sum_{1 \leq i \leq N} \phi_{1}\left(X_{i}\right)$, assuming that $\mathbb{E} \phi_{1}(X)=0$, $\mathbb{E} \varphi(x, X)=0$ for all $x \in \mathfrak{X}, \mathbb{E} \phi^{2}(x, X)<\infty, \mathbb{E}_{1}^{2}(X)<\infty$. We will provide bounds for $\Delta_{N}=\sup _{x} \mid F(x)$ $-F_{0}(x)-F_{1}(x) \mid$, where $F$ is a distribution function of $T$ and $F_{0}, F_{1}$ are its limiting distribution function and Edgeworth correction respectively. Applications of these results are also provided for von Mises statistics case.

Keywords: U-Statistics, Von Mises Statistics, Symmetric Statistics

\section{Introduction}

Consider the measurable space $(\mathfrak{X}, \mathfrak{B}, \mu)$, with measure $\mu=\mathcal{L}(\mathfrak{X})$. Let $L=L^{2}(\mathfrak{X}, \mathfrak{B}, \mu)$ denote the real Hilbert space of square integrable real functions. Let $\mathbb{Q}: L^{2} \rightarrow L^{2}$ denote the Hilbert-Schmidt operator associated with the kernel $\phi$ and defined via

$$
\mathbb{Q} f(x)=\int_{X} \phi(x, y) f(y) \mu(\mathrm{d} y)=\mathbb{E} \phi(x, X) f(X),
$$

Let $q_{1}, q_{2} \cdots$ be its eigenvalues. Without loss of generality we shall assume that $\left|q_{1}\right| \geq\left|q_{2}\right| \geq \cdots$.

Let $\left\{e_{j}: j \geq 1\right\}$ denote an orthonormal complete system of eigenfunctions of $\mathbb{Q}$ of the corresponding eigenvalues $q_{1}, q_{2} \cdots$. Then

$$
\sigma^{2}=\mathbb{E} \phi^{2}(\bar{X}, X)=\sum_{j \geq 1} q_{j}^{2}, \phi(x, y)=\sum_{j \geq 1} q_{j} e_{j}(x) e_{j}(y)
$$

since $\mathbb{Q}$ is a Hilbert-Schmidt operator and the kernel $\phi$ is degenerate. The series in (1.1) converges in $L^{2}$ $\left(\mathfrak{X}^{2}, \mathfrak{B}^{2}, \mu \times \mu\right)$. Consider the subspace $L^{2}\left(\phi, \phi_{1}\right) \subset L^{2}$ $\left(\mathfrak{X}^{2}, \mathfrak{B}^{2}, \mu \times \mu\right)$ generated by $\phi_{1}$ and eigenfunctions $e_{j}$ corresponding to nonzero eigenvalues $q_{j}$. Introducing, if necessary, an eigenvalues $e_{0}: \mathbb{Q} e_{0}=0$,we can assume that $e_{0}, e_{1}, \cdots$ is an orthonormal basis in $L^{2}(\phi$, $\left.\phi_{1}\right)$. Thus, we have

$$
\phi_{1}(x)=\sum_{j \geq 0} a_{j} e_{j}(x) \text { in } L_{2}, \quad \beta_{2}=\mathbb{E} \phi_{1}^{2}(X)=\sum_{j \geq 0} a_{j}^{2},
$$

with $a_{j}=\mathbb{E} \phi_{1}(X) e_{j}(X)$ and $\mathbb{E} e_{j}(X)=0$, for all $j$. Therefore $\left(e_{j}(X)\right)_{j \geq 0}$ is an orthonormal system of random variables with zero means.

Hilbert space $\ell_{2} \subset \mathbb{R}^{\infty}$ consists of $x=\left(x_{1}, x_{2} \cdots\right) \in$ $\mathbb{R}^{\infty}$, such that

$$
|x|^{2}=_{\text {def }}\langle x, x\rangle,\langle x, y\rangle=\sum_{j \geq 0} x_{j} y_{j},|x|<\infty .
$$

Consider the random vector

$$
X={ }_{\text {def }}\left(e_{0}(X), e_{1}(X), e_{2}(X), \cdots\right),
$$

which takes values in $\mathbb{R}^{\infty}$. Since $\left(e_{j}(X)\right)_{j \geq 0}$ is a system of mean zero uncorrelated random variables with variances 1 , the random vector $X$ has mean zero and $\operatorname{cov}\left(e_{i}, e_{j}\right)=\delta_{i j}$ and $\delta_{i j}$ is Kronecker's symbol. Using (1.1) and (1.2), we can write

$$
\phi(X, \bar{X})=\langle\mathbb{Q} X, X\rangle, \phi_{1}(X)=\langle a, X\rangle,
$$

where we define $\mathbb{Q} x=\left(0, q_{1} X_{1}, q_{2} X_{2}, \cdots\right)$ for $x \in \mathbb{R}^{\infty}$ and $a=\left(a_{j}\right)_{j \geq 0} \in \mathbb{R}^{\infty}$. The equalities (1.4) allow us to assume that the measurable space $\mathfrak{X}$ is $\mathbb{R}^{\infty}$. Let $X$ 
be a random vector taking values in $\mathbb{R}^{\infty}$ with mean zero and covariance $\operatorname{cov}\left(X_{i}, X_{j}\right)=\delta_{i j}$ and that

$$
\phi(X, \bar{X})=\langle\mathbb{Q} X, \bar{X}\rangle, \phi_{1}(X)=\langle a, X\rangle .
$$

Without loss of generality we shall assume that the kernels $\phi(x, y)$ and $\phi_{1}(x)$ are linear functions in each of their arguments $([2])$.

Introduce the definitions:

$$
\begin{gathered}
\beta_{s}=\mathbb{E}\left|\phi_{1}(X)\right|^{s}, \quad \gamma_{s}=\mathbb{E}|\phi(X, \bar{X})|^{s}, \quad \sigma^{2}=\gamma_{2}, \\
\gamma_{s, r}=\mathbb{E}\left(\mathbb{E}\left\{|\phi(X, \bar{X})|^{s} \mid X\right\}\right)^{r},
\end{gathered}
$$

and assume that

$$
\beta_{2}<\infty, 0<\sigma^{2}<\infty .
$$

for the statistic $T$ we can write

$$
\mathbb{E} T^{2}=\beta_{2}+\frac{N-1}{2 N} \sigma^{2} .
$$

The statistic $T$ is called degenerate since $\sigma^{2}>0$ ensures that the quadratic part of the statistic is not asymptotically negligible and therefore statistic $T$ is not asymptotically normal. More precisely, the asymptotic distribution of $T$ is non-Gaussian and is given by the distribution of the random variable

$$
T_{0}=\frac{1}{2} \sum_{j \geq 1} q_{j}\left(\eta_{j}^{2}-1\right)+\sum_{j \geq 0} a_{j} \eta_{j},
$$

$\eta_{j}$ is a sequence of i.i.d. standard normal variables, $a_{0}, a_{1}, \cdots$ denotes a sequence of square summable weights and $\left|q_{1}\right| \geq\left|q_{2}\right| \geq \cdots$ denote eigenvalues of the Hilbert-Schmidt operator, say $\mathbb{Q}$, associated with the kernel $\phi$.

Consider the concentration functions of statistic $T_{*}$

$$
\begin{aligned}
& Q\left(T_{*} ; \lambda\right)=\sup _{x} \mathbb{P}\left\{x \leq T_{*} \leq x+\lambda\right\}, \lambda \geq 0, \\
& T_{*}=\sum_{1 \leq i<k \leq N} \varphi\left(X_{j}, X_{k}\right)+f_{1}\left(X_{1}, \cdots, X_{M}\right) \\
& \quad+f_{2}\left(X_{M+1}, \cdots, X_{N}\right),
\end{aligned}
$$

where $f_{1}=f_{1}\left(X_{1}, \cdots, X_{M}\right)$ is an arbitrary statistic depending only on $X_{1}, \cdots, X_{M}, f_{2}=f_{2}\left(X_{M+1}, \cdots, X_{N}\right)$ is as well arbitrary but independent of $X_{1}, \cdots, X_{M}$. Note that the class of statistics $T_{*}$ is slightly more general than the class of statistics $T$. We shall denote $c, c_{1}, \cdots$ constants. If a constant depends on, say s, we shall write $c(s)=c_{s}$.

Consider the distibution functions

$$
\begin{gathered}
F(x)=\mathbb{P}\{T \leq x\}, \quad F_{0}(x)=\mathbb{P}\left\{T_{0} \leq x\right\}, \\
\Delta_{N}=\sup _{x}\left|\Delta_{N}(x)\right|, \quad \Delta_{N}(x)=F(x)-F_{0}(x)-F_{1}(x),
\end{gathered}
$$

$F_{1}(x)$ denotes an Edgeworth correction. The Edgeworth correction $F_{1}(x)=F_{1}\left(x ; \mathcal{L}(X), \phi_{1}, \phi\right)$ is defined as a function of bounded variation satisfying $F_{1}(-\infty)=0$ and with the Fourier-Stieltjes transform given by

$$
\hat{F}_{1}(t)=\frac{(i t)^{3}}{6 \sqrt{N}} \mathbb{E}\left(\phi_{1}(X)+\phi(X, G)\right)^{3} e\left\{t T_{0}\right\} .
$$

Let us notice that $F_{1}$ vanishes if $\phi_{1}=0$ or if

$$
\begin{aligned}
\mathbb{E} \phi_{1}^{3}(X) & =\mathbb{E} \phi_{1}^{2}(X) \phi(X, x)=\mathbb{E} \phi_{1}(X) \phi^{2}(X, x) \\
& =\mathbb{E} \phi^{3}(X, x)=0,
\end{aligned}
$$

holds for all $x \in \mathfrak{X}$. Using the technique presented in this work we may obtain the result for approximation bound of order $O\left(N^{-1}\right)$ for U-statistic distribution function which has an order $\left|q_{9}\right|^{-\alpha}$ (see Theorem 3, 2) below) or $\left|q_{13}\right|^{-\alpha}$ (see Theorem 3, 1) below) with respect to dependence on first nine or thirteen eigenvalues of operator $\mathbb{Q}$, respectively.

\section{Auxiliary Results}

Consider the vector $G=\left(\eta_{0}, \eta_{1} \cdots\right)$ with values in $\mathbb{R}^{\infty}$, where $\eta_{0}, \eta_{1}, \cdots$ standard normal variables. Let us formulate lemma in which equalities for the moments of determinants of random matrices consisting of the scalar products such as $\left\langle\mathbb{Q} G_{i}, G_{j}\right\rangle$ are obtained. Analogue of this lemma is proved in [1] for matrices consisting of the scalar products such as $\left\langle G_{i}, G_{j}\right\rangle$ where $G$-Gaussian (0, $\left.\sigma^{2} \mathbb{I}\right)$ vector.

Lemma 1. Let $G_{1}, \cdots, G_{s}, G_{1}^{\prime}, \cdots, G_{s}^{\prime}$ be random elements in a Hilbert space $H$ such that $G_{i}=\left(\eta_{0}, \eta_{1} \cdots\right)$, where $\eta_{0}, \eta_{1}, \cdots$ standard normal variables. Let $\left|q_{1}\right|$ $\geq\left|q_{2}\right| \geq \cdots$ be the eigenvalues of Hilbert-Schmidt operator $\mathbb{Q} . W=(\operatorname{det} \mathbb{A})^{2}$, where $\mathbb{A}=\mathbb{A}(G)=\left\{a_{i j}(G)\right\}_{i, j=1}^{s}$, $a_{i j}(G)=\phi\left(G_{i}, G_{j}^{\prime}\right)=\left\langle\mathbb{Q} G_{i}, G_{j}\right\rangle$.

Then

$$
\mathbb{E} W=(s !)^{2} \sum_{1 \leq i_{1}<\ldots<i_{s}<\infty}\left(q_{i_{1}} \ldots q_{i_{s}}\right)^{2},\left(\mathbb{E} W^{2}\right)^{1 / 2} \leq c(s) \mathbb{E} W .
$$

\section{Nondegeneracy condition}

We shall assume that random vector $Z$, a kernel $\phi$, parameters $c, c_{1}, s$ and $p$ satisfy the nondegeneracy condition if

$$
\begin{gathered}
\mathbb{P}\{W(\bar{Z})>\delta\} \geq p, \quad \delta=q_{1}^{2} \ldots q_{9}^{2}, \\
\mathbb{P}\left\{\left|\phi\left(Z_{i}, \bar{Z}_{j}\right)\right| \leq c\right\} \geq c_{1}, \quad 1 \leq i, \quad j \leq s,
\end{gathered}
$$

where $W(\bar{Z})=(\operatorname{det} A)^{2}, \quad A=\left\{a_{i j}\right\}_{i, j=1}^{s}, a_{i j}=\varphi\left(Z_{i}, \bar{Z}_{j}\right)$, $Z_{i}, \bar{Z}_{j}$ are independent copies of $Z$.

Here parameter $p$ is small and parameter $c_{1}$ is close to 1 . Let $\mathcal{N}(\delta, p)$ denote the set of all vectors $Z$ satisfying the nondegeneracy condition.

Notice that $G$ satisfies the nondegeneracy condition. Let vectors $G$ and $X$ have equal means and covari- 
ances, then

$$
\begin{gathered}
\mathbb{E} \phi_{1}(G)=\mathbb{E} \phi(G, x)=0, \mathbb{E} \phi_{1}^{2}(G)=\mathbb{E} \phi_{1}^{2}(X), \\
\mathbb{E} \phi_{1}(G) \phi(G, x)=\mathbb{E} \phi_{1}(X) \phi(X, x), \\
\mathbb{E} \phi(G, x) \varphi(G, y)=\mathbb{E} \phi(X, x) \phi(X, y) .
\end{gathered}
$$

The following Lemma 2 means that increase of $n$ yields equivalence of nondegeneracy conditions fulfillments for sum and Gaussian vector.

Lemma 2. Let $G \in \mathcal{N}\left(4 q_{1}^{2} \cdots q_{9}^{2}, 1-p\right)$ be a Gaussian random vector and $\mathbb{P}\left\{W(\bar{G})>4 q_{1}^{2} \cdots q_{9}^{2}\right\} \geq 1-p$. Then for $m \geq c_{s}\left|q_{1} \cdots q_{9}\right|^{-3} p^{-1}\left(\left|q_{1} \cdots q_{9}\right|^{-3} p^{-1} \gamma_{2,3 / 2}+\gamma_{3}\right)$ we have $S_{m} \in \mathcal{N}\left(q_{1}^{2} \cdots q_{9}^{2}, 1-2 p\right)$, where $S_{m}=m^{-1 / 2}\left(X_{1}+\right.$ $\left.\cdots+X_{m}\right)$ is random sum.

Further, it is necessary to bound the characteristic function of the statistic $T_{*}$. That will be done in Lemmas 3, 4 and Theorem 1.

The following Lemma 3 has a similar proof to Lemma 6.5 from [2].

By $\tau, \tau_{1}, \tau_{2} \cdots$ we shall denote independent copies of a symmetric random variable $\tau$ with nonnegative characteristic function and such that

$$
1 \leq \mathbb{E} \tau^{2} \leq 2, \mathbb{P}\{|\tau| \leq 2\}=1 .
$$

Lemma 3. Let $s \in \mathbb{N}$ and $L \in \mathbb{Z}_{+}$. Assume that vector $Y \in \mathcal{N}\left(\left(q_{1} \cdots q_{9}\right)^{2}, p\right)$ takes values in $R^{\infty}$. Write

$$
\Lambda=\sum_{j=1}^{s L} \tau_{j} Y_{j}, \quad \bar{\Lambda}=\sum_{j=1}^{s L} \bar{\tau}_{j} \bar{Y}_{j}, \quad q=[p L / 4],
$$

where $Y_{j}$ and $\bar{Y}_{j}$ are independent copies of $Y$. Then

$$
\begin{gathered}
\mathbb{E} e\left\{t \phi(\Lambda, \bar{\Lambda}\} \leq c_{d}(s)(p L)^{-d}+\sup _{\mathbb{A}} \mathbb{E} e\{t\langle\mathbb{A} U, V\rangle\},\right. \\
t \in \mathbb{R}, d \geq 0,
\end{gathered}
$$

where $\sup _{\mathbb{A}}$ denotes the supremum over all $s \times s$ nonrandom matrices $\mathbb{A}$ such that $(\operatorname{det} \mathbb{A})^{2}>q_{1}^{2} \cdots q_{9}^{2}$.

$U$ and $V$ denote independent vectors in $\mathbb{R}^{s}$ which are sums of $n$ independent copies of $W=\left(\tau_{1}, \cdots, \tau_{s}\right)$.

In the following lemma the bound from above for the characteristic function $\mathbb{E} e\{t\langle\mathbb{A} U, V\rangle\}$ is received. This results was proved in [1]. The received estimation contains the determinant of matrix in right-hand side of inequality. This fact allows to use eigenvalues of operator $\mathbb{Q}$ for the estimation of characteristic function.

Lemma 4. Let $A$ be a nondegenerate $s \times s$ matrix. Let $X \in \mathbb{R}^{s}$ denote a random vector with covariance $C$. Assume that there exists a constant $c_{s}$ such that

$$
\mathbb{P}\left\{|X| \leq c_{s}\right\}=1,|A| \leq c_{s},\left|C^{-1}\right| \leq c_{s} .
$$

Let $U$ and $V$ denote independent random vectors which are sums of $n$ independent copies of $X$. Then

$$
|\mathbb{E} e\{t\langle A U, V\rangle\}| \leq c(s)|\operatorname{det} A|^{-1} \mathcal{M}^{2 s}(t ; N) \text { for }|t|>0,
$$

where $\mathcal{M}(t ; N)=1 / \sqrt{|t| N}+\sqrt{|t|}$ for $|t|>0$.

Using our Lemmas 3 and 4 we may obtain a bound for characteristic function for statistic $T_{*}$.

Theorem 1. Let $m \in \mathbb{N}$. Assume that the sum $T=(2 m)^{-1 / 2}\left(\tilde{X}_{1}+\cdots+\tilde{X}_{m}\right) \in \mathcal{N}\left(\left(q_{1} \cdots q_{9}\right)^{2}, p\right)$. Then, for any statistic $T_{*}$ we have

$$
\left|\mathbb{E} e\left\{t T_{*}\right\}\right| \ll_{s} \frac{1}{\left|q_{9}\right|^{9}} \mathcal{M}^{2 s}(t m ; p M / m) .
$$

The proof of this theorem is similar to proof of Theorem 6.2 in [2].

Write :

$$
\psi(t)=\left|\mathbb{E}_{9} e\left\{t T^{9}\right\}\right| .
$$

In following lemma a multiplicative inequality for characteristic function of $T^{9}$ is given. This inequality yields the desired bound $\mathcal{O}\left(N^{-1}\right)$ for an integral of the characteristic function of a $U$-statistic. Similar result was proved in Lemma 7.1 in [2]

Lemma 5. Let $d \geq 0$ and $s \in \mathbb{N}$. Assume that $Y=(2 m)^{-1} \sum_{k=1}^{k=m} \tilde{X}_{k} \in \mathcal{N}\left(\left(q_{1} \cdots q_{9}\right)^{2}, p\right)$. Then there exist constants $c_{1}(s, d)$ and $c_{2}(s, d)$ such that the event

$$
D=\left\{\psi(t-\gamma) \psi(t+\gamma) \leq c_{1}(s, d) \frac{1}{\left|q_{9}\right|^{9} v} \mathcal{M}^{s}(\gamma m ; p M / m)\right\},
$$

satisfies

$$
\mathbb{P}\{D\} \geq 1-c_{2}(s, d)(p M / m)^{-d} .
$$

For $A \geq t_{0}, t_{1} \geq 0$ define the integrals

$$
I_{0}=\int_{-t_{1}}^{t_{1}}|\hat{\Psi}(t)| \mathrm{d} t, \quad I_{1}=\int_{t_{0} \leq t \mid \leq A}|\hat{\Psi}(t)| \frac{\mathrm{d} t}{|t|},
$$

where $\hat{\Psi}=\int_{\mathbb{R}} e\{t x\} \mathrm{d} \Psi(x)$ denotes the Fourier-Stieltjes transform of the distribution function $\Psi(x)=\mathbb{P}\left\{T_{*} \leq x\right\}$. The estimation for these integrals is received in following lemma, which has a proof similar to Lemma 3.3 in [2].

Lemma 6. Let $m \in \mathbb{N}$. Assume that the random vector $Y=(2 m)^{-1 / 2}\left(\tilde{X}_{1}+\ldots+\tilde{X}_{m}\right) \in \mathcal{N}\left(\left(q_{1} \cdots q_{9}\right)^{2}, p\right)$ and $s \geq 9$. Let

$$
\begin{gathered}
k=\frac{p M}{m}, t_{0}=\frac{c_{0}(s)}{m} k^{-1+2 / s}, t_{1}=\frac{c_{1}(s)}{m} k^{-1 / 2}, \\
\frac{c_{2}(s)}{m} \leq A \leq \frac{c_{3}(s)}{m},
\end{gathered}
$$

where $c_{j}(s), 0 \leq j \leq 3$ are some positive constants.

Then

$$
I_{0} \ll_{s}\left|q_{9}\right|^{-9}(p M)^{-1}, \quad I_{1} \ll_{s} \max \left\{1,\left|q_{9}\right|^{-18}\right\} m(p M)^{-1} .
$$




\section{Approximation Accuracy Estimation}

For $r \in \mathbb{Z}_{+}$and functions $f_{i}$, introduce the statistic

$$
T^{(r)}=\frac{1}{N} \sum_{1 \leq i<j \leq N} \phi\left(Z_{i}, Z_{j}\right)+\sum_{1 \leq i \leq N} f_{i}\left(Z_{i}\right)
$$

where

$$
Z_{j}=X_{j} \text { for } 1 \leq j \leq r, Z_{j}=G_{j} \text { for } r<j \leq N .
$$

Write $l=[(N-2) / 20]$ and put

$$
\kappa(t)=\kappa(t, N, \phi, \mathcal{L}(X))=\kappa_{1}(t)+\kappa_{2}(t),
$$

where

$$
\begin{gathered}
\kappa_{1}(t)=\sup _{L}\left|\mathbb{E} e\left\{t N^{-1} \sum_{1 \leq j<k \leq l} \phi\left(X_{j}, X_{k}\right)+L\left(X_{1}, \cdots, X_{l}\right)\right\}\right|, \\
\kappa_{2}(t)=\sup _{L}\left|\mathbb{E} e\left\{t N^{-1} \sum_{1 \leq j<k \leq l} \phi\left(G_{j}, G_{k}\right)+L\left(G_{1}, \cdots, G_{l}\right)\right\}\right|,
\end{gathered}
$$

where supremum is taken over all linear statistics $L$, that is, over all functions which can be represented as $L\left(x_{1}, \cdots, x_{l}\right)=\sum_{j=1}^{l} f_{j}\left(x_{j}\right)$ with some functions $f_{1}, \cdots$, $f_{l}$.

Consider the following Lemma 7, which has a similar proof as Lemma 4.2 in [2].

Lemma 7. Let $m \in \mathbb{N}, s \geq 9$ and $t_{0}=m^{-1}(p N /$ $m)^{-1+2 / s}$. Assume that the random vector $Y=(2 m)^{-1 / 2}$ $\left(\tilde{X}_{1}+\cdots+\tilde{X}_{m}\right)$ satisfies the nondegeneracy condition. Then, for $p N>m, m^{-1} \geq t_{*} \geq t_{0}$ the distribution function $F^{(r)}$ of $T^{(r)}$ satisfies

$$
F^{(r)}(x)=\frac{1}{2}+\frac{i}{2 \pi} V \cdot P \cdot \int_{-N t_{*}}^{N t_{*}} e\{-x t\} \hat{F}^{(r)}(t) \frac{\mathrm{d} t}{t}+R,
$$

where $|R| \ll_{s}\left(\left|q_{9}\right|^{-9}+\max \left\{1,\left|q_{9}\right|^{-18}\right\}\right) m /(p N)$.

The Edgeworth correction $F_{1}(x)=F_{1}\left(x ; \mathcal{L}(X), \phi_{1}, \phi\right)$ is defined as a function of bounded variation satisfying $F_{1}(-\infty)=0$ and with the Fourier-Stieltjes transform given by

$$
\hat{F}_{1}(t)=\frac{(i t)^{3}}{6 \sqrt{N}} \mathbb{E}\left(\phi_{1}(X)+\phi(X, G)\right)^{3} e\left\{t T_{0}\right\} .
$$

Lemma 8. Assume that the nondegeneracy condition is fulfilled.

1) Let $s \geq 13$ and $m_{0} \asymp\left|q_{1} \cdots q_{9}\right|^{-3} p^{-1}\left(\left|q_{1} \cdots q_{9}\right|^{-3}\right.$ $\left.p^{-1} \gamma_{2,3 / 2}+\gamma_{3}\right)$. Then

$$
\begin{aligned}
\Delta_{N} \ll_{s} & \frac{m_{0}\left(\left|q_{9}\right|^{-9}+\max \left\{1,\left|q_{9}\right|^{-18}\right\}\right)}{p N} \\
& +N^{-1}\left(\beta_{3}^{2}+\sigma^{2} \gamma_{2,2}\right)\left(\frac{1}{\left|q_{s}\right|^{s}}+\frac{1}{\left|q_{s}\right|^{6}}\right)+\frac{\left|q_{9}\right|^{-9}}{p^{6} N} \\
& \cdot\left(\beta_{4}+\beta_{3}^{2}+\sigma^{2}+\gamma_{3}+\sigma^{2} \gamma_{3}+\gamma_{2,2}+\sigma^{2} \gamma_{2,2}\right)
\end{aligned}
$$

2) Assume that the condition (1.8) holds and that $s \geq 9$. Then

$$
\begin{aligned}
\Delta_{N} \ll_{s} & \frac{m_{0}\left(\left|q_{9}\right|^{-9}+\max \left\{1,\left|q_{9}\right|^{-18}\right\}\right)}{p N} \\
& +N^{-1}\left(\beta_{3}^{2}+\sigma^{2} \gamma_{2,2}\right)\left(\frac{1}{\left|q_{s}\right|^{s}}+\frac{1}{\left|q_{s}\right|^{6}}\right)+\frac{\left|q_{9}\right|^{-9}}{p^{4} N} \\
& \cdot\left(\beta_{4}+\sigma^{2}+\gamma_{3}+\gamma_{2,2}\right) .
\end{aligned}
$$

To prove this lemma we need to make the same steps as in Lemma 4.1 in [2] replacing Theorem 6.2 by Theorem 1.

Now we can formulate a following Theorem 2, where bounds for $\Delta_{N}$ are obtained. This theorem were proved in [4]:

Theorem 2. 1) Let $s \geq 13$

$m_{0} \asymp\left|q_{1} \cdots q_{9}\right|^{-3} p^{-1}\left(\left|q_{1} \cdots q_{9}\right|^{-3} p^{-1} \gamma_{2,3 / 2}+\gamma_{3}\right), p_{0} \asymp c(s)$. Then

$$
\begin{aligned}
\Delta_{N} \ll & \frac{m_{0}\left(\left|q_{9}\right|^{-9}+\max \left\{1,\left|q_{9}\right|^{-18}\right\}\right)}{c N} \\
& +N^{-1}\left(\beta_{3}^{2}+\sigma^{2} \gamma_{2,2}\right)\left(\frac{1}{\left|q_{13}\right|^{13}}+\frac{1}{\left|q_{13}\right|^{6}}\right)+\frac{\left|q_{9}\right|^{-9}}{c N} \\
& \cdot\left(\beta_{4}+\beta_{3}^{2}+\sigma^{2}+\gamma_{3}+\sigma^{2} \gamma_{3}+\gamma_{2,2}+\sigma^{2} \gamma_{2,2}\right),
\end{aligned}
$$

2) Assume that (1.8) holds and $s \geq 9$. Then

$$
\begin{aligned}
\Delta_{N} \ll & \frac{m_{0}\left(\left|q_{9}\right|^{-9}+\max \left\{1,\left|q_{9}\right|^{-18}\right\}\right)}{c N} \\
& +N^{-1}\left(\beta_{3}^{2}+\sigma^{2} \gamma_{2,2}\right)\left(\frac{1}{\left|q_{9}\right|^{9}}+\frac{1}{\left|q_{9}\right|^{6}}\right)+\frac{\left|q_{9}\right|^{-9}}{c N} \\
& \cdot\left(\beta_{4}+\sigma^{2}+\gamma_{3}+\gamma_{2,2}\right) .
\end{aligned}
$$

\section{An Extension of Bounds to Von Mises Statistics. Applications}

Assuming that the kernels $\phi$ and $\phi_{1}$ are degenerate, consider the von Mises statistic

$$
M=\frac{1}{2 N} \sum_{1<i, j<N} \phi\left(X_{i}, X_{j}\right)+\frac{1}{\sqrt{N}} \sum_{1 \leq i \leq N} \phi_{1}\left(X_{i}\right) .
$$

Introducing the function $\psi(x)=(\phi(x, x)-v) / 2$ with $v=E \phi(X, X)$, we can rewrite (4.1) as

$$
M-\frac{v}{2}=T+\frac{1}{N} \sum_{1 \leq i \leq N} \psi\left(X_{i}\right)
$$

In this section we shall extend the bounds to statistics of type (4.2), assuming that $E \psi(X)=0$ and $\rho=E \psi^{2}$ $(X)<\infty$.

Similarly to the case of $T$, we can represent the kernel $\phi$ (respectively, $\phi_{1}$ and $\psi$ ) as a bilinear (respec- 
tively, linear) function, defined on $\mathrm{R}^{\infty}$. However in this case we have to assume that $\mathrm{R}^{\infty}$ has an additional coordinate since $\psi$ can be linearly independent of $\phi_{1}$ and of the eigenfunctions of $\mathbb{Q}$. To fix notation, we shall assume that $\mathrm{R}^{\infty}$ consists of vectors $x=\left(x_{-1}, x_{0}\right.$, $\left.x_{1}, \cdots\right)$. Then all representations and results of Section 2 concerning $\phi$ and $\phi_{1}$ still hold, and for $\psi$ we have $\psi(x)=\langle b, x\rangle$ with some $b=\left(b_{-1}, b_{0}, b_{1}, \cdots\right)$ such that $\sum_{j \geq-1} b_{j}^{2}<\infty$. Write $\psi_{0}(x)=\sum_{j \geq 0} b_{j} x_{j}$.

Introduce the function $F_{*}$ of bounded variation (provided that $q_{3} \neq 0$ ) with the Fourier-Stieltjes transform

$$
\hat{F}_{*}(t)=\frac{i t}{\sqrt{N}} E \psi(G) e\left\{t T_{0}\right\}=\frac{i t}{\sqrt{N}} E \psi_{0}(G) e\left\{t T_{0}\right\}
$$

and such that $F_{*}(-\infty)=0$. Bellow we shall show that (see Lemma 9.3 [2])

$$
\hat{F}_{*}(t)=\frac{(i t)^{2}}{\sqrt{N}} E \psi(X)\left(\varphi_{1}(X)+\varphi(X, G)\right) e\left\{t T_{0}\right\} .
$$

Notice that $F_{*}=0$ whenever $\phi_{1}=0$.

Write $H_{1}=F_{1}+F_{*}$, and let $H$ denote the distribution function of $M-v / 2$. Define

$$
\delta_{N}=\sup _{x}\left|\delta_{N}(x)\right|, \quad \delta_{N}(x)=H(x)-F_{0}(x)-H_{1}(x) .
$$

Theorem 3.1) Assume that $q_{13} \neq 0$. Then we have

$$
\begin{aligned}
\delta_{N} \leq & \frac{m_{0}\left(\left|q_{9}\right|^{-9}+\max \left\{1,\left|q_{9}\right|^{-18}\right\}\right)}{c N} \\
& +N^{-1}\left(\beta_{3}^{2}+\sigma^{2} \gamma_{2,2}\right)\left(\frac{1}{\left|q_{13}\right|^{13}}+\frac{1}{\left|q_{13}\right|^{6}}\right)+\frac{\left|q_{9}\right|^{-9}}{c N} \\
& \cdot\left(\beta_{4}+\sigma^{2}+\gamma_{3}+\gamma_{2,2}+\rho\right) .
\end{aligned}
$$

2) Assume that (1.8) is fulfilled and $q_{9} \neq 0$. Then we have

$$
\begin{aligned}
\Delta_{N} \ll & \frac{m_{0}\left(\left|q_{9}\right|^{-9}+\max \left\{1,\left|q_{9}\right|^{-18}\right\}\right)}{c N} \\
& +N^{-1}\left(\beta_{3}^{2}+\sigma^{2} \gamma_{2,2}\right)\left(\frac{1}{\left|q_{9}\right|^{9}}+\frac{1}{\left|q_{9}\right|^{6}}\right)+\frac{\left|q_{9}\right|^{-9}}{c N} \\
& \cdot\left(\beta_{4}+\sigma^{2}+\gamma_{3}+\gamma_{2,2}+\rho\right) .
\end{aligned}
$$

Proof. We shall use the following estimates. Write

$$
\xi=\frac{t}{N} \sum_{1 \leq j \leq N} \psi\left(X_{j}\right), \quad \zeta=\frac{t}{N} \sum_{1 \leq j \leq N} \psi\left(G_{j}\right) .
$$

Expanding with remainder $\mathcal{O}(\xi)$, splitting the sum $\xi$ in parts and conditioning, we have

$$
|E \mathrm{e}\{t T+\xi\}-E \mathrm{e}\{t T\}-i E \xi \mathrm{e}\{t T\}| \ll \chi N^{-1} t^{2} \rho .
$$

Proceeding similarly to the proof of Lemma 8.2 from [2], we obtain

$$
\left|\hat{F}_{*}(t)-i E \zeta \mathrm{e}\left\{R_{[1, N]} T\right\}\right| \ll \chi N^{-1} t^{2}\left(\rho+\sigma^{2}\right) .
$$

Applying the Bergstrom-type identity

$$
\begin{aligned}
& \mathbb{E} S=\mathbb{E} \mathcal{R}_{[1, N]} S+\sum_{j=1}^{N}\left\{\mathbb{E} \mathcal{R}_{[2, j]} S-\mathbb{E} \mathcal{R}_{[1, j]} S\right\}, \\
& \mathcal{R}_{[1, j]} S=S\left(G_{1}, \cdots, G_{j}, X_{j+1}, \cdots, X_{N}\right)
\end{aligned}
$$

with $S=\xi \mathrm{e}\{t T\}$ and proceeding similarly to the proof of Lemma 8.3 from [2], we get

$$
\begin{aligned}
& \left|E \xi \mathrm{e}\{t T\}-E \zeta \mathrm{e}\left\{\mathcal{R}_{[1, N]} T\right\}\right| \\
& \ll \chi N^{-1}\left(t^{2}+t^{4}\right)\left(\rho+\beta_{4}+\beta_{3}^{2}+\gamma_{3}+\gamma_{2,2}+\gamma_{2} \Gamma_{2,2}\right) .
\end{aligned}
$$

Arguments similar to the proof of Lemma 8.5 from [2] allow proving

$$
\left|\hat{F}_{*}(t)\right| \ll N^{-1.2}|t| \rho^{1 / 2} \prod_{j \leq 1}\left(1+2 t^{2} q_{j}^{2} / 25\right)^{-1 / 4},
$$

and, for $s \geq 3$,

$$
\begin{gathered}
\int_{|t| \geq \lambda}\left|\hat{F}_{*}(t)\right| \frac{\mathrm{d} t}{|t|} \ll_{s} N^{-1 / 2} \rho^{1 / 2}\left|q_{s}\right|^{-s / 2} \lambda^{1-s / 2}, \lambda>0 \\
\int_{\mathrm{R}}\left|\hat{F}_{*}(t)\right| \frac{\mathrm{d} t}{|t|} \ll_{s} N^{-1 / 2} \rho^{1 / 2}\left|q_{s}\right|^{-1}
\end{gathered}
$$

The estimates (4.6)-(4.11) allow proceeding similarly to the proof of Theorem 2, using a lemma similar to Lemma 8. Proving such a lemma, we have to apply Lemma 8 to the distribution function $H$. This is possible since that statistic $M-v / 2$ is a statistic of type (3.1). The estimates (4.10) and (4.11) allow application of the Fourier inversion to the function $F_{*}$. As a result, we arrive at

$$
\int_{-N t_{*}}^{N t_{*}}\left|\hat{H}(t)-\hat{F}_{0}(t)-\hat{H}_{1}(t)\right| \frac{\mathrm{d} t}{|t|} .
$$

Here, however, we have $\hat{H}(t)=E \mathrm{e}\{t T+\xi\}$, and

$$
\begin{aligned}
& \left|\hat{H}(t)-\hat{F}_{0}(t)-\hat{H}_{1}(t)\right| \leq\left|\hat{F}(t)-\hat{F}_{0}(t)-\hat{F}_{1}(t)\right| \\
+ & |E \mathrm{e}\{t T+\xi\}-E \mathrm{e}\{t T\}-i E \xi \mathrm{e}\{t T\}| \\
+ & \left|\hat{F}_{*}(t)-i E \zeta \mathrm{e}\left\{R_{[1, N]} T\right\}\right| \\
+ & +E \xi \mathrm{e}\{t T\}-E \zeta \mathrm{e}\left\{R_{[1, N]} T\right\} \mid .
\end{aligned}
$$

Therefore, using (4.6)-(4.8), we can proceed as in the proof of Lemma 11. As a final result we get bounds similar to those of Theorem 2, with the additional summand $\rho$.

\section{References}

[1] V. Ulyanov and F. Götze, "Uniform Approximations in the CLT for Balls in Euclidian Spaces," 00-034, SFB 343, University of Bielefeld, 2000, p. 26. 
http://www.math.uni-bielfeld.de/sfb343/preprints/pr00034. pdf.gz

[2] V. Bentkus and F. Götze, "Optimal Bounds in NonGaussian Limit Theorems for U-Statistics," The Annals of Probability, Vol. 27, No.1, 1999, pp. 454-521. doi:10.1214/aop/1022677269

[3] S. A. Bogatyrev, F. Götze and V. V. Ulyanov, "NonUniform Bounds for Short Asymptotic Expansions in the CLT for Balls in a Hilbert Space," Journal of Multivari- ate Analysis, Vol. 97, 2006, pp. 2041-2056.

[4] T. A. Zubayraev, "Asymptotic Analysis for U-Statistics: Approximation Accuracy Estimation," Publications of Junior Scientists of Faculty of Computational Mathematics and Cybernetics, Moscow State University, Vol. 7, 2010, pp. 99-108.

http://smu.cs.msu.su/conferences/sbornik7/smu-sbornik-7 .pdf 